\section{RMD Open}

Rheumatic \&

Musculoskeletal Diseases

\title{
Long-term patient survival in a Swedish population-based cohort of patients with ANCA-associated vasculitis
}

\author{
Caroline Heijl, ${ }^{1}$ Aladdin J Mohammad, ${ }^{2}$ Kerstin Westman, ${ }^{1}$ Peter Höglund ${ }^{3}$
}

To cite: Heijl C, Mohammad AJ, Westman $\mathrm{K}$, et al. Long-term patient survival in a Swedish population-based cohort of patients with ANCA-associated vasculitis. RMD Open 2017;3:e000435. doi:10.1136/ rmdopen-2017-000435

Received 16 January 2017 Revised 3 May 2017 Accepted 5 May 2017

\section{(a) CrossMark}

${ }^{1}$ Department of Clinical Sciences Lund, Division of Nephrology, Lund University, Lund, Sweden ${ }^{2}$ Department of Clinical Sciences Lund, Section of Rheumatology, Lund University, Lund, Sweden and Department of Renal Medicine, Vasculitis and lupus Clinic, Addenbrooke's Hospital, Cambridge, UK

${ }^{3}$ Department of Laboratory Medicine, Division of Clinical Chemistry and Pharmacology, Lund University, Lund, Sweden

Correspondence to

Dr Caroline Heijl; Caroline.Heij|@ med.lu.se

\section{ABSTRACT}

Objectives Patients with antineutrophil cytoplasmatic antibodies-associated vasculitides (AAV) exhibit higher mortality than the general population. In the current study, we assessed whether cluster affiliation based on clinical presentation might predict mortality.

Methods With case record review, the outcomes for a population-based cohort of patients diagnosed with AAV in southern Sweden (catchment area of 0.7 million inhabitants) between 1997 and 2010 were assessed. Based on organ involvement at presentation, the cohort was stratified into the following clusters: gastrointestinal, cardiovascular, non-renal, renal with proteinase 3 (PR3) and renal without PR3. Cluster affiliation, demographics, clinical and laboratory values at entry were tested as prognostic factors for survival in multivariable models. Results 195 patients (98 female) with a median age of 69 years (IQR 55-77) at diagnosis were included in the cohort. The median time of follow-up was 4 years for the 98 patients (50\%) who died during follow-up and 11 years for those alive at end of follow-up. The 1-year, 2-year, 5 -year and 10 -year survival was $87 \%, 82 \%, 70 \%$ and $55 \%$, respectively. Prognostic factors for survival were sex, age, renal function and cluster affiliation. The mortality of patients with AAV was significantly increased compared with the general population except in the non-renal cluster. The cardiovascular and gastrointestinal clusters showed the highest mortality.

Conclusion Even though the mortality in patients with AAV is increased compared with the general population this does not apply to patients without gastrointestinal, cardiovascular or renal involvement at diagnosis. We

suggest that the initial clinical presentation is an important predictor for survival.

\section{INTRODUCTION}

Antineutrophil cytoplasmatic antibodies (ANCA)-associated vasculitides (AAV) is a group of life-threatening diseases with unclear aetiology. The diseases consist of three disease phenotypes: granulomatosis with polyangiitis (GPA), microscopic polyangiitis (MPA) and eosinophilic granulomatosis with polyangiitis (EGPA) that all are characterised by inflammation in small vessels and in most cases presence of antibodies against neutrophil

\section{Key messages}

What is already known about this subject?

- The mortality in patients with antineutrophil cytoplasmatic antibodies (ANCA)-associated vasculitis (AAV) is increased compared with the general population.

- Older age, renal impairment, inflammatory activity, accumulated damage of the disease hypoalbuminaemia, cardiomyopathy and preexisting comorbidities are all proposed risk factors for mortality in patients with AAV.

What does this study add?

- Results from follow-up studies of patients with AAV participating in clinical trials are applicable to patients with $A A V$ in ordinary practice.

- The use of cluster affiliation as a classification tool in assessing the long-term outcome for patients with AAV is validated for the first time.

- The mortality in patients with AAV still is increased compared with the general population, but this does not apply to patients without gastrointestinal, cardiovascular or renal involvement at presentation.

How might this impact on clinical practice?

- Cluster affiliation constitutes a new approach to the prediction of mortality risk in individual patients with AAV. The model may easily be adopted and enable the treating physicians to inform their patients in a way that may be easily understood.

granulae proteins (proteinase 3 (PR3) or myeloperoxidase). ${ }^{1}$ The main organs affected are the kidneys, lungs, ear-nose-throat and the skin but the symptoms and signs might vary and the diseases are often elusive and therefore a substantial amount of time might elapse before the diagnosis is established. ${ }^{2}$ Without treatment, the survival is poor, with less than $80 \%$ surviving the first year. ${ }^{3}$ The introduction of immunosuppressive treatment has improved the survival but mortality is still increased compared with the general population. ${ }^{4}$ We have previously assessed 
the long-term outcome and predictors of death in patients with AAV, who had participated in one of four randomised trials organised by the European Vasculitis Study Group (EUVAS). ${ }^{6}$ In a randomised trial, there are several exclusion and inclusion criteria that have to be taken into account before generalising the results. ${ }^{7}$ Even though the EUVAS studies included a broad spectrum of patients with AAV, they excluded patients with EGPA, very elderly patients, those with life-threatening lung haemorrhage at the time of diagnosis and those who had a known previous malignancy.

The AAV diagnoses are heterogenic, with many overlapping features between GPA, MPA and EGPA, a fact that has recently led to the suggestion of using cluster analysis in the categorising of the diseases. ${ }^{8}$ Patients $(n=715)$ with AAV who had participated in clinical trials (the EUVAS long-term follow-up study and the French WEGENT study $^{6-13}$ ) were grouped together in five different clusters based on their clinical similarities at presentation and the cluster affiliation correlated better with survival than the traditional way of dividing the AAV-group into the diagnoses of GPA, MPA or EGPA.

Recognising the consequences of selection of patients in clinical trials, our aim in the present study is to assess the long-term survival and predictors of death in a population-based cohort of patients with AAV without any referral or exclusion bias.

We also aim to validate cluster affiliation as an additional parameter in the survival analysis as previously described by Mahr et al. ${ }^{8}$

\section{METHODS}

\section{Study design and participants}

The outcomes for all incident cases with suspected AAV in a catchment area of approximately 0.7 million inhabitants in southern Sweden between 1997 and 2010 were assessed. All diagnoses were verified by case record review according to a predefined criteria described previously, ${ }^{14}$ and patients were followed from time of presentation until death or 31 December 2015. Patients were classified into the three disease categories, GPA, MPA and EGPA, according to the European Medicines Agency algorithm. ${ }^{15}$ Since we wanted to include all consecutive patients, we decided to include also patients who had participated in the EUVAS trials reported in the longterm follow-up. ${ }^{6}$ Causes of death were evaluated by review of case records when available, and the remaining were retrieved from the Causes of Death Register.

The study was done in accordance with the principles of the Declaration of Helsinki and was approved by the Ethical Review Board in Lund, Sweden (2010-517).

\section{Baseline evaluation and treatment}

Results from laboratory investigations at diagnosis included plasma creatinine, full blood count, platelets, $\mathrm{C}$ reactive protein and ANCA serotype analysed with ELISA and capture-ELISA. According to then current guidelines used in Southern Sweden, the majority of patients received cyclophosphamide and glucocorticoids as induction treatment. Patients with creatinine $>500$ $\mathrm{mol} / \mathrm{L}$ (rapidly progressive glomerulonephritis) or pulmonary haemorrhage received plasma exchange and or intravenous methylprednisolone in addition to cyclophosphamide as induction treatment. For those without systemic involvement, that is, exclusively upper respiratory involvement, induction therapy was methotrexate together with corticosteroids. As an alternative to cyclophosphamide, from 2007, rituximab has been used in a few patients for induction of remission. After stable remission, the treatment was continued with azathioprine in the majority of patients.

\section{Statistical analysis}

The cases were divided into five clusters (A-E), based on clinical presentation at diagnosis, as described by Mahr et $a l^{8}$ and were gastrointestinal (E), cardiovascular (D), non-renal (A), renal with PR3 (B) and renal without PR3 (C) characteristics.

Continuous variables are presented as medians with IQRs. Categorical variables are expressed as counts and frequencies. Survival was calculated using the KaplanMeier method. The log-rank test was used to compare the overall patient survival with that of the Swedish general population matched for age, sex and calendar year, calculated with the Hakulinen method. ${ }^{16}$ Similar analyses were also performed for each cluster. We decided in advance to include the following parameters in a multivariable Cox regression analysis: sex, age, diagnosis, ANCA serotype and estimated glomerular filtration rate divided into chronic kidney disease (CKD) groups (1-2, 3, 4 and 5, respectively). ${ }^{17}$ In addition, we performed a Cox regression analysis using the variables, sex, age and cluster. In these analyses, the proportional hazards assumption was tested by the weighted scaled Schoenfield residuals test. If the proportional hazards assumption was not met, we constructed time-varying coefficients as described by Thomas and Reyes. ${ }^{18}$

\section{RESULTS \\ Demography}

One hundred and ninety-five patients (98 female) with a median age of 69 years (55-77) at diagnosis fulfilled the inclusion criteria and were included in the study cohort. Among these, 94 (48\%) were diagnosed with GPA, 90 with MPA (46\%) and $11(6 \%)$ with EGPA. Diagnoses were biopsy proven in 172 patients and all were verified by case record review. Further demographic data are summarised in table 1 . Of the patients included in this cohort, seven had also participated in the EUVAS longterm follow-up study, ${ }^{6}$ four in the CYCLOPS study ${ }^{11}$ and three in the MEPEX study. ${ }^{12}$

Cluster affiliation and baseline characteristics are given in table 1. Clusters A-C are based on renal involvement and ANCA serotype and are without cardiovascular or 


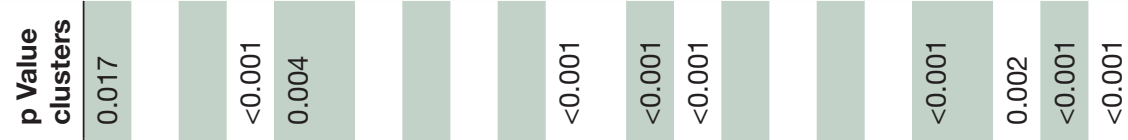

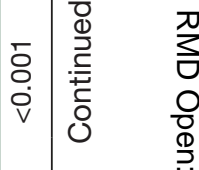

可

.

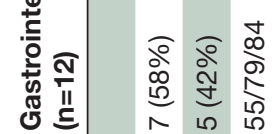

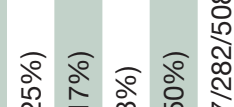

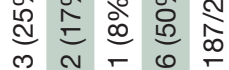

న్

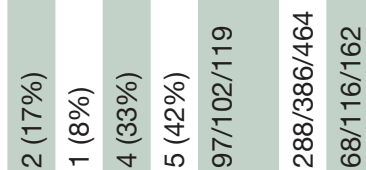

ลे จे จे

过

离

\begin{tabular}{c} 
a \\
$\frac{0}{0}$ \\
$\frac{\pi}{0}$ \\
$\frac{1}{0}$ \\
0 \\
0 \\
\hline
\end{tabular}

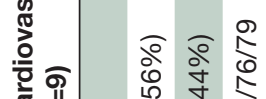

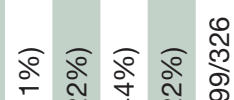

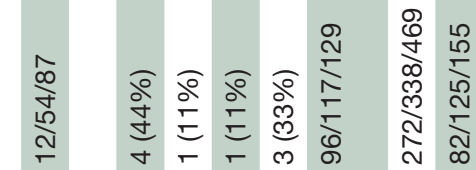

ㅇำ

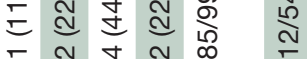

ल्ల ल्ల ल्ల్ల

똠

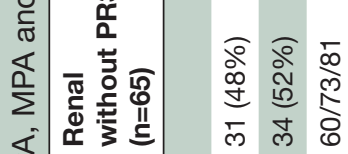

วะ $\bar{\circ}$

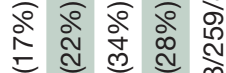

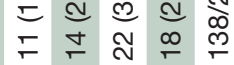

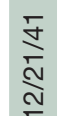

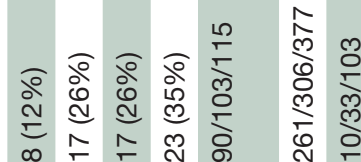

वेo

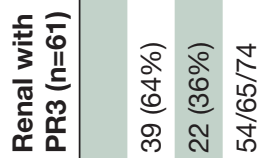

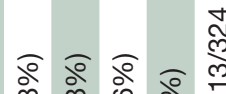

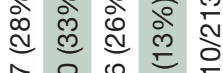

$\stackrel{2}{\circ}$
$\stackrel{0}{7}$

वे वे वे वे

oै

孚

난

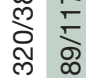

ㅇำ

$\begin{array}{lll}\overline{0} & 0 & 0 \\ ㅇ & 0 & 0\end{array}$

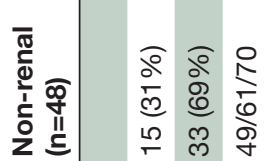

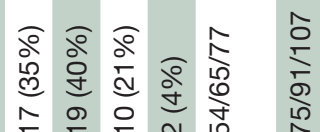

ल 0

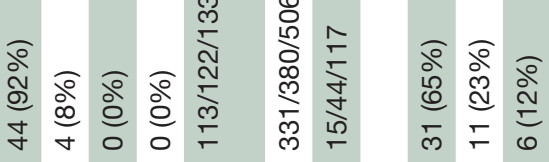

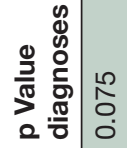

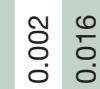

$\begin{array}{lll}\bar{o} & \bar{\sigma} & \overline{0} \\ \dot{0} & 0 & 0 \\ \dot{v} & 0\end{array}$

$\begin{array}{llll}\overline{0} & \overline{0} & \overline{0} & \overline{0} \\ 0 & 0 & 0 \\ \dot{v} & 0 & 0 & 0\end{array}$

$\overline{8}$
$\dot{v}$

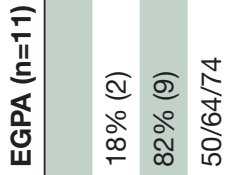

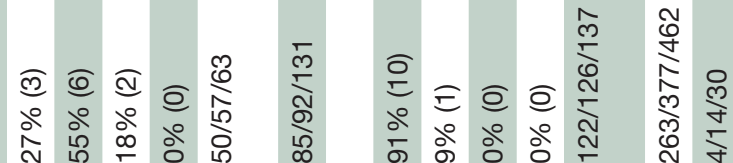

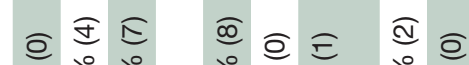
ஃे @े

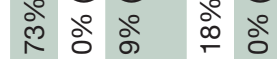

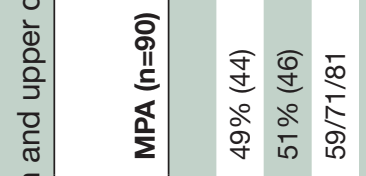

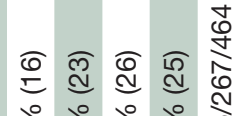

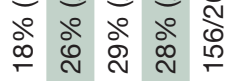

앙

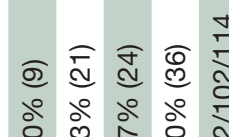

足

$-2$

(1)

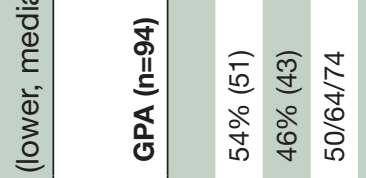

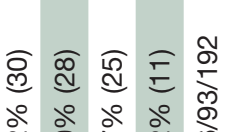

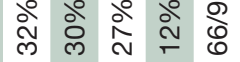

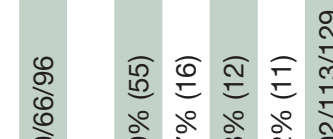

営

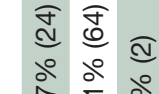

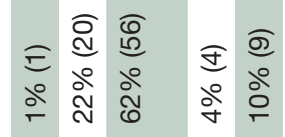

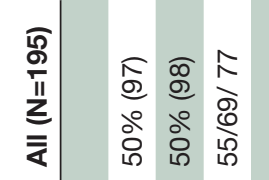

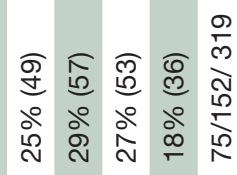

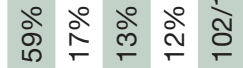

$\overbrace{\substack{\infty \\ \infty}}^{\infty}$

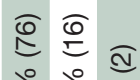

बु $\widehat{\text { ¿ }}$ 잉 $\stackrel{\circ}{\circ}$

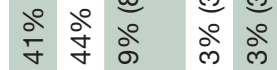

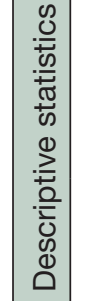

弪 号

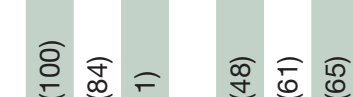

के 今े

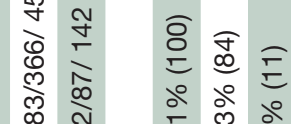

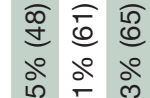

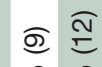

फ

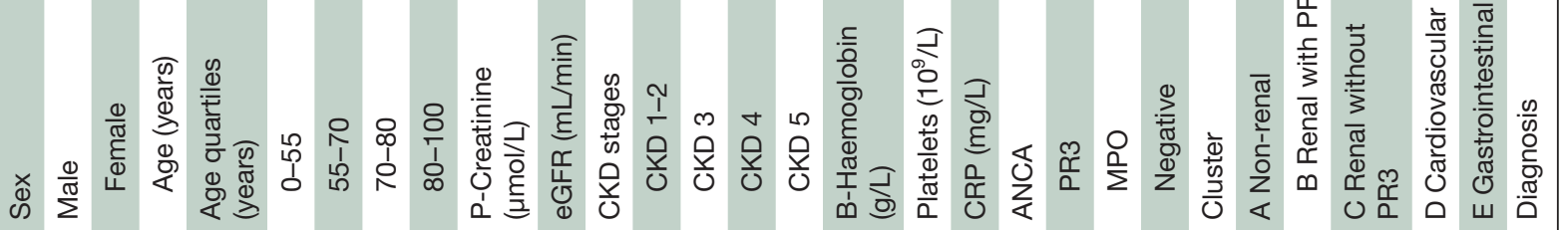


gastrointestinal symptoms. In the cardiovascular disease group, the symptoms were cardiomyopathy $(n=1)$, heart failure $(n=2)$, pericarditis $(n=1)$, pericardial fluid $(n=1)$, myocardial infarction $(\mathrm{n}=2)$ and new-onset atrial fibrillation $(n=1)$. In the gastrointestinal disease group, the symptoms were severe abdominal pain $(n=6)$, bloody diarrhoea $(n=2)$, pancreatitis $(n=1)$ and gut perforation $(\mathrm{n}=1)$.

\section{Survival}

The time of follow-up ranged between 2.3 and 18 years with a median time of 11 years for those who were alive at the end of 2015 and a median time of 4 years for patients who died during follow-up (range $0.3-17$ years). The 1-year, 2-year, 5-year and 10-year survival in the present study was $87 \%, 82 \%, 70 \%$ and $55 \%$, respectively. In the AAV cohort, there was a significant increased mortality compared with the age-matched, sex-matched and calendar year-matched general population (figure 1). We observed 98 deaths but only 40 were expected in the general population $(\mathrm{p}<0.001, \log$-rank test). The survival for each cluster and their matched general population are presented in figure 2. For each of the clusters (A-E), the observed number of deaths were $12,30,40,8$ and 8 , respectively. The expected number of deaths in these clusters was 10.0, 11.0, 16.5, 0.8 and 2.2, respectively. All comparisons were significant $(p<0.001)$, except for the non-renal cluster $(p=0.527)$. The risk ratio for observed over expected deaths in cluster A was 1.20 (95\% CI 0.65 to 1.90 , exact binomial test).

Predictors of death: multivariable and cluster analysis

Kaplan-Meier plots for survival by sex showed an initial higher mortality for men compared with women, but

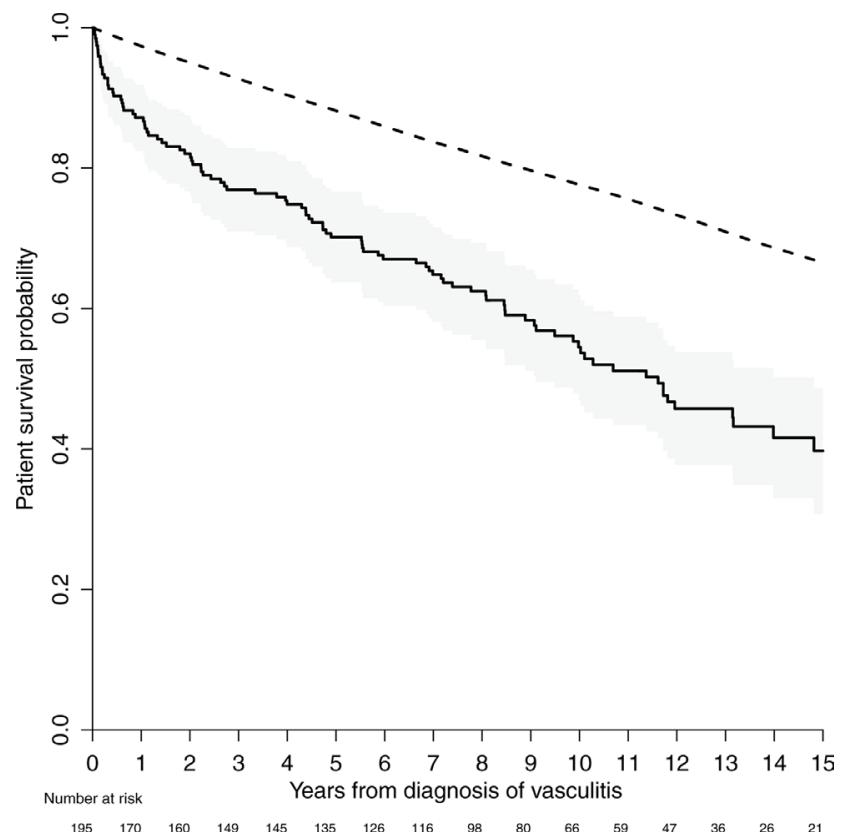

Figure 1 Patient survival overall (solid line) with $95 \% \mathrm{Cl}$ (grey area) compared with a sex-matched, age-matched and calendar year-matched general population (dashed line). 


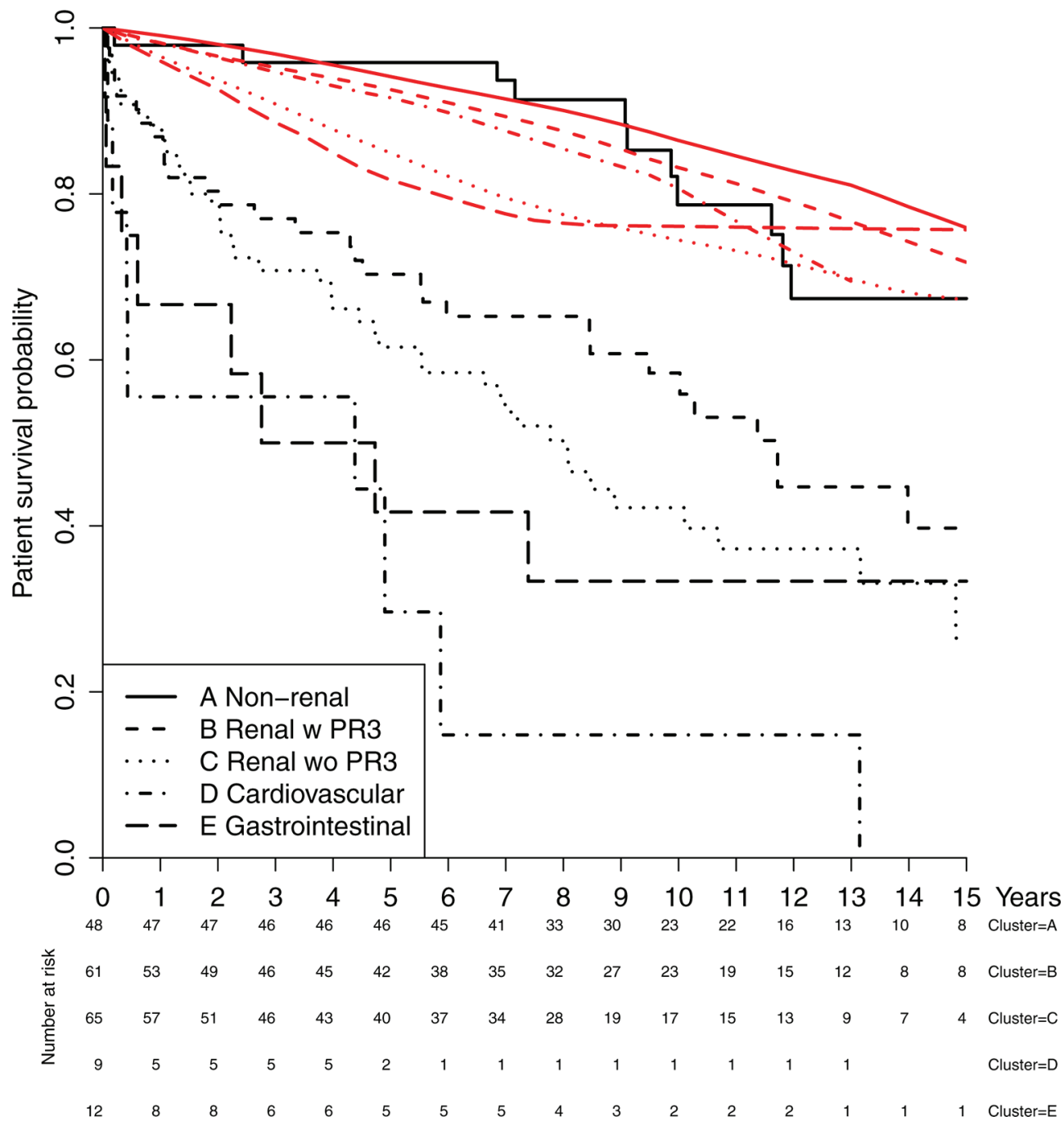

Figure 2 Patient survival divided into clusters (black lines), each compared with a sex-matched, age-matched and calendar year-matched general population (red lines).

the two survival curves crossed at approximately 13 years (data not shown). As could be suspected from this plot, the proportional hazards assumption of a Cox model including sex was not fulfilled for this variable. Instead, when we divided the effects from sex into three strata $((0-1.8),(1.8-8.5)$ and greater than 8.5 years) we were able to fulfil this criterion. Consequently, we used these strata in the analyses. Significant predictors of death evaluated with our first multivariable Cox regression analysis were advancing age, gender, CKD group 5, but not diagnosis or ANCA serotype (table 2). Compared with the CKD group 1-2, groups 3-5 had higher HRs, but only group 5 reached significance. In our second multivariable Cox regression analysis, advancing age, gender and cluster affiliation (table 3) were significant predictors.

\section{Causes of death}

A total of 98 deaths occurred during follow-up (50\%). Of these, 66 cases $(67 \%)$ were evaluated with case record review but the remaining 32 cases $(33 \%)$ died unattended at home and the causes of death were only available through the death certificates in 26 cases, but in eight cases, with deaths occurring after the latest date of update for this registry, the death had to be classified as unknowns (table 4). The category miscellaneous in table 4 was used for five reported causes of death: respiratory failure in two patients, suicide, hepatic failure and arteriovenous fistula bleeding. Twenty-seven (28\%) of the 98 deaths occurred during the first year after diagnosis with the main causes of death being active vasculitis in seven patients (six male and one female) and bacterial infection in five. After the first year of follow-up, the main causes of death were malignancy (14), bacterial infection (15) and cardiovascular disease in another 14 patients.

\section{DISCUSSION}

This study, done in a population-based cohort of patients including all three diagnoses within the AAV spectrum, shows that patient survival is decreased compared with a matched general population. In addition, this is the first validation of the cluster affiliation model proposed by Mahr et al. ${ }^{8}$ Predictors of death in this study were increased age, decreased renal function 
Table 2 HR of death in patients with AAV across subgroups identified by multivariable Cox regression

\begin{tabular}{|c|c|c|c|c|}
\hline & HR & $\begin{array}{l}\text { LCL } \\
\text { HR }\end{array}$ & $\begin{array}{l}\text { UCL } \\
\text { HR }\end{array}$ & $p$ Value \\
\hline Female & 1 & & & (reference) \\
\hline Male (0-1.85years) & 2.04 & 0.99 & 4.20 & 0.053 \\
\hline Male (1.85-8.5 years) & 0.90 & 0.49 & 1.67 & 0.747 \\
\hline Male (>8.5years) & 0.35 & 0.13 & 0.94 & 0.036 \\
\hline Age (1-year increase) & 1.09 & 1.07 & 1.12 & $<0.001$ \\
\hline Diagnosis GPA & 1 & & & (reference) \\
\hline Diagnosis EGPA & 0.60 & 0.09 & 4.14 & 0.606 \\
\hline Diagnosis MPA & 1.40 & 0.80 & 2.44 & 0.234 \\
\hline ANCA PR3 & 1 & & & (reference) \\
\hline ANCA MPO & 0.94 & 0.57 & 1.54 & 0.807 \\
\hline ANCA negative & 2.24 & 0.46 & 11.00 & 0.320 \\
\hline CKD 1-2 & 1 & & & (reference) \\
\hline CKD 3 & 1.14 & 0.56 & 2.36 & 0.713 \\
\hline CKD 4 & 1.75 & 0.89 & 3.45 & 0.108 \\
\hline CKD 5 & 2.51 & 1.30 & 4.83 & 0.006 \\
\hline
\end{tabular}

AAV, ANCA-associated vasculitides; ANCA, antineutrophil cytoplasmatic antibodies; CKD, chronic kidney disease; EGPA, eosinophilic granulomatosis with polyangiitis; GPA, granulomatosis with polyangiitis; LCL, lower confidence limit; MPA, microscopic granulomatosis; MPO, myeloperoxidase; PR3, proteinase 3; UCL; upper confidence limit.

Table 3 HR of death in patients with AAV across subgroups identified by cluster affiliation, multivariable Cox regression

\begin{tabular}{lllll}
\hline & HR & $\begin{array}{l}\text { LCL } \\
\text { HR }\end{array}$ & $\begin{array}{l}\text { UCL } \\
\text { HR }\end{array}$ & p Value \\
\hline Female & 1 & & & (reference) \\
Male (0-1.85years) & 1.89 & 0.92 & 3.90 & 0.084 \\
Male (1.85-8.5years) & 0.84 & 0.46 & 1.55 & 0.582 \\
Male (>8.5 years) & 0.34 & 0.13 & 0.90 & 0.029 \\
Age (1-year increase) & 1.09 & 1.07 & 1.12 & $<0.001$ \\
Cluster A, non-renal & 1 & & & (reference) \\
Cluster B, renal with PR3 & 2.53 & 1.25 & 5.12 & 0.010 \\
\hline $\begin{array}{l}\text { Cluster C, renal without } \\
\text { PR3 }\end{array}$ & 2.70 & 1.34 & 5.46 & 0.005 \\
Cluster D, cardiovascular & 6.38 & 2.52 & 16.15 & $<0.001$ \\
Cluster E, gastrointestinal & 3.98 & 1.52 & 10.39 & 0.005 \\
\hline
\end{tabular}

Cluster affiliation for AAV (Mahr et $\left.\mathrm{al}^{8}\right)$ with nine clinical variables and ANCA specificity.

AAV, ANCA-associated vasculitis; ANCA, antineutrophil cytoplasmatic antibody; LCL, lower confidence limit; PR3 proteinase 3; UCL; upper confidence limit.

and cluster affiliation with cluster affiliation as the strongest predictor.

Our survival results are consistent with previous studies both within the EUVAS clinical trials and others. ${ }^{519-21}$
The 1-year, 2-year, 5-year and 10-year survival in the present study was $87 \%, 82 \%, 70 \%$ and $55 \%$, respectively. In comparison the 1-year, 2-year and 5-year survival in the EUVAS follow-up cohort was $88 \%, 85 \%$ and $78 \%$, respectively. ${ }^{6}$ As previously shown, the mortality is high during the first year after diagnosis, and consistent with the results from the EUVAS follow-up cohort, the mortality risk was higher in younger patients when compared with an age-matched and gender-matched general population. The main specific causes of death during this period were active vasculitis and infections. We observed a higher mortality in men during the first 1.85 years of follow-up and a higher mortality in women after 8.5 years of follow-up. We noted that of the seven deaths from vasculitis during the first 1.85 years, six were male and only one female, suggesting either more severe disease, not sufficiently aggressive immunosuppression or longer delay between first symptoms and contact with the healthcare system in males compared with females. The lower incidence of fatal infections during the first year may reflect the possibility of a more individualised immunosuppressive treatment in patients outside the protocol of a clinical trial. In elderly patients, the doses of immunosuppressive treatment may be lower and more individualised compared with a protocol of a clinical trial. The median age of the patients in this cohort was 69 years, compared with 61 years in the EUVAS trials. ${ }^{9-12}$ After the first year, the main causes of death were malignancy, bacterial infection and cardiovascular disease, which is consistent with the EUVAS long-term follow-up study. ${ }^{6}$

The subcategorisation of AAV into clusters (cardiovascular, gastrointestinal, non-renal, renal with PR3 and renal without PR3) stratifies the patients in different categories according to initial clinical presentation. Patients within the non-renal cluster do not exhibit any statistically significant difference in mortality compared with the general population. This is in contrast to all the other clusters. The highest mortality risk is seen in the cardiovascular and the gastrointestinal clusters. The increased mortality in patients with $\mathrm{AAV}$ with cardiovascular disease is also highlighted in a previous study from Hazebroek $e t$ $a l^{22}$

The use of cluster analysis of patients with AAV has recently been described within the EUVAS study group and our results further validate the prognostic relevance of this stratification. ${ }^{8}$ We observed very similar results in terms of mortality after having divided our cohort of patients into the five different clusters previously reported in a larger group of patients. This emphasises the suggestion that AAV can be seen as a phenotypic continuum and that the initial clinical presentation may be better suited to subgroup of the patients than traditional diagnoses and ANCA subtypes. The different clusters are mixes of patients diagnosed with GPA, MPA or EGPA. Patients with EGPA were not included in the previous study. ${ }^{8}$ In our study, patients with EGPA are represented in three of 
Table 4 Causes of death

Time from diagnosis to death

\begin{tabular}{lllllll}
\hline & $\mathbf{0 - 1 . 8 5}$ years & \multicolumn{2}{l}{$\mathbf{1 . 8 5 - 8 . 5}$ years } & > 8.5years \\
\cline { 2 - 7 } Causes of death & Female & Male & Female & Male & Female & Male \\
\hline Vasculitis & 1 & 6 & 0 & 0 & 1 & 0 \\
Renal failure & 2 & 4 & 2 & 1 & 1 & 0 \\
Bacterial infection & 2 & 3 & 5 & 5 & 2 & 3 \\
\hline Pneumocystis & 1 & 0 & 0 & 0 & 1 & 0 \\
\hline Cardiovascular & 3 & 4 & 5 & 5 & 3 & 2 \\
\hline Malignancy & 0 & 4 & 7 & 2 & 1 & 1 \\
Gastrointestinal & 0 & 1 & 1 & 2 & 0 & 0 \\
Dementia & 0 & 0 & 1 & 1 & 2 & 0 \\
Miscellaneous & 0 & 1 & 0 & 2 & 2 & 0 \\
Unknown & 2 & 0 & 2 & 2 & 2 & 0 \\
\hline Total & 11 & 23 & 23 & 20 & 15 & 6 \\
\hline
\end{tabular}

the clusters (table 1), suggesting that this category might also be incorporated into further cluster analysis of AAV.

The main strengths of this study are that it investigates a well-characterised, population-based cohort including patients with all types of AAV. In a randomised trial, there are several exclusion and inclusion criteria that have to be taken into account before generalising the results to individual patients. ${ }^{7}$ Even though the EUVAS study included a broad spectrum of patients with AAV, it excluded patients with EGPA, very elderly patients, those with life-threatening lung haemorrhage at the time of diagnosis and those who had a known previous malignancy. In the present study, we show that the findings in the EUVAS long-term follow-up study are applicable in a population-based cohort of patients, treated according to current treatment protocols. The length of follow-up (median 11 years for survivors) is longer than in most other studies investigating the survival in AAV, adding strength to our results. In addition, the present study validates a new way of partitioning AAV into clusters as well as comparing the survival in the different clusters with an age-matched and gender-matched general population.

The main weakness is that it might be difficult to implement the results from this cohort that includes all types of AAV into individual patients with a specific diagnosis (ie, GPA, MPA or EGPA) but on the other hand, cluster affiliation might be a better way to predict mortality in patients with AAV than traditional diagnoses. Another, at least potential weakness is that we have not used Birmingham Vasculitis Activity Score $(\mathrm{BVAS})^{23}$ as a predictor, as we did in the EUVAS longterm follow-up study and that has also been used in other studies. ${ }^{24}$ In clinical trials or in other settings where patients are evaluated and followed according to protocol, BVAS may be of greater use compared with a pure clinical setting, with physicians who may not be trained in using this scale. In addition, this material has been collected since 1997, when BVAS was not fully implemented in routine practise in our region. Further, in a retrospective study using medical records as source for BVAS scoring, the quality of data would most probably be poor.

Since malignancies are common causes of morbidity and death in the long-term follow-up in this study as well as in others, ${ }^{25} 26$ a suggestion for a future study is to investigate the malignancy risk in these patients.

In conclusion, in this cohort of patients with AAV, we show that the results from follow-up studies of patients with AAV participating in clinical trials are applicable to patients in ordinary practice. Furthermore, this study validates and strengthens the proposed cluster affiliation as an additional tool in assessing the long-term outcome for patients with AAV, demonstrating that the initial clinical presentation might be a more important predictor for survival than diagnosis and type of ANCA. Finally, we show that, even though the mortality in patients with AAV still is increased compared with the general population, this does not apply to the patients in the non-renal cluster.

Contributors $\mathrm{CH}$ provided the initial idea for the study, designed the study, collected data, scrutinised medical records, interpreted data, performed statistical analyses, designed the tables and figures and wrote the first draft and subsequent iterations of the manuscript. AJM initiated and coordinated the AAV cohort used in this study, ascertained diagnosis and classification of AAV, collected data at baseline and contributed to data collection at follow-up and reviewed and provided critical comments on drafts. KW designed the study, interpreted data, designed the tables and figures and reviewed and provided critical comments on drafts. PH designed the study, interpreted data, performed statistical analyses, designed the tables and figures and reviewed and provided critical comments on the manuscript.

Funding This study has been funded by grants from Region Skåne, that is, the healthcare provider of the catchment area for the patients and the Swedish Rheumatism Association (Reumatikerförbundet). The sponsors had no role in the study design or data analysis and interpretation; or in the decision to submit the manuscript for publication. The corresponding author had full access to all the data and had final responsibility for the decision to submit for publication.

Competing interests None declared.

Ethics approval The Ethical Review Board in Lund, Sweden (2010-517). 
Provenance and peer review Not commissioned; externally peer reviewed. Data sharing statement There are no additional data to share.

Open Access This is an Open Access article distributed in accordance with the Creative Commons Attribution Non Commercial (CC BY-NC 4.0) license, which permits others to distribute, remix, adapt, build upon this work non-commercially, and license their derivative works on different terms, provided the original work is properly cited and the use is non-commercial. See: http://creativecommons.org/ licenses/by-nc/4.0/

(C) Article author(s) (or their employer(s) unless otherwise stated in the text of the article) 2017. All rights reserved. No commercial use is permitted unless otherwise expressly granted.

\section{REFERENCES}

1. Jennette JC, Falk RJ, Bacon PA, et al. 2012 revised international chapel hill consensus conference nomenclature of vasculitides. Wiley Subscription Services, Inc. A Wiley Company, 20122013:1-11.

2. Mohammad AJ, Jacobsson LT, Mahr AD, et al. Prevalence of Wegener's granulomatosis, microscopic polyangiitis, polyarteritis nodosa and Churg-Strauss syndrome within a defined population in Southern Sweden. Rheumatology 2007;46:1329-37.

3. Fauci AS, Wolff SM, Johnson JS. Effect of cyclophosphamide upon the immune response in wegener's granulomatosis. $N$ Engl J Med 1971;285:1493-6.

4. Mohammad AJ, Jacobsson LT, Westman KW, et al. Incidence and survival rates in wegener's granulomatosis, microscopic polyangiitis, churg-strauss syndrome and polyarteritis nodosa. Rheumatology 2009;48:1560-5.

5. Hilhorst $\mathrm{M}$, Wilde $\mathrm{B}$, van Paassen $\mathrm{P}$, et al. Improved outcome in anti-neutrophil cytoplasmic antibody (ANCA)-associated glomerulonephritis: a 30-year follow-up study. Nephrol Dial Transplant 2013;28:373-9.

6. Flossmann O, Berden A, de Groot K, et al. Long-term patient survival in ANCA-associated vasculitis. Ann Rheum Dis 2011;70:488-94.

7. Pagnoux C, Carette S, Khalidi NA, et al. Comparability of patients with ANCA-associated vasculitis enrolled in clinical trials or in observational cohorts. Clin Exp Rheumatol 2015;33:77-83.

8. Mahr A, Katsahian S, Varet H, et al. Revisiting the classification of clinical phenotypes of anti-neutrophil cytoplasmic antibodyassociated vasculitis: a cluster analysis. Ann Rheum Dis 2013;72:1003-10.

9. De Groot K, Rasmussen N, Bacon PA, et al. Randomized trial of cyclophosphamide versus methotrexate for induction of remission in early systemic antineutrophil cytoplasmic antibody-associated vasculitis. Arthritis Rheum 2005;52:2461-9.

10. Jayne D, Rasmussen N, Andrassy K, et al. A randomized trial of maintenance therapy for vasculitis associated with antineutrophil cytoplasmic autoantibodies. N Engl J Med 2003;349:36-44.
11. de Groot K, Harper L, Jayne DR, et al. Pulse versus daily oral cyclophosphamide for induction of remission in antineutrophil cytoplasmic antibody-associated vasculitis: a randomized trial. Ann Intern Med 2009;150:670-80.

12. Jayne DR, Gaskin G, Rasmussen N, et al. Randomized trial of plasma exchange or high-dosage methylprednisolone as adjunctive therapy for severe renal vasculitis. J Am Soc Nephrol 2007;18:2180-8.

13. Pagnoux C, Mahr A, Hamidou MA, et al. Azathioprine or methotrexate maintenance for ANCA-associated vasculitis. $N$ Engl $J$ Med 2008;359:2790-803.

14. Mohammad AJ, Jacobsson LT, Mahr AD, et al. Prevalence of Wegener's granulomatosis, microscopic polyangiitis, polyarteritis nodosa and Churg-Strauss syndrome within a defined population in Southern Sweden. Rheumatology 2007;46:1329-37.

15. Watts R, Lane S, Hanslik T, et al. Development and validation of a consensus methodology for the classification of the ANCAassociated vasculitides and polyarteritis nodosa for epidemiological studies. Ann Rheum Dis 2007;66:222-7.

16. Hakulinen T. Cancer survival corrected for heterogeneity in patient withdrawal. Biometrics 1982;38:933-42.

17. Webster AC, Nagler EV, Morton RL, et al. Chronic kidney disease. Lancet 2016.

18. Thomas L, Reyes EM. Tutorial: survival estimation for cox regression models with time-varying coefficients using SAS and $R$. J Stat Softw 2014:61:1-23.

19. Holle JU, Gross WL, Latza U, et al. Improved outcome in 445 patients with Wegener's granulomatosis in a German vasculitis center over four decades. Arthritis Rheum 2011;63:257-66.

20. Puéchal X, Pagnoux C, Perrodeau É, et al. Long-term outcomes among participants in the WEGENT trial of remission-maintenance therapy for granulomatosis with polyangiitis (Wegener's) or microscopic polyangiitis. Arthritis Rheumatol 2016;68:690-701.

21. Hruskova Z, Casian AL, Konopasek $P$, et al. Long-term outcome of severe alveolar haemorrhage in ANCA-associated vasculitis: a retrospective cohort study. Scand J Rheumatol 2013;42:211-4.

22. Hazebroek MR, Kemna MJ, Schalla S, et al. Prevalence and prognostic relevance of cardiac involvement in ANCA-associated vasculitis: eosinophilic granulomatosis with polyangiitis and granulomatosis with polyangiitis. Int J Cardiol 2015;199:170-9.

23. Luqmani RA, Bacon PA, Moots RJ, et al. Birmingham Vasculitis Activity Score (BVAS) in systemic necrotizing vasculitis. QJM 1994;87:671-8.

24. Ritchie J, Reynolds T, Robson JC. Long-Term Outcome of ANCAAssociated Systemic Vasculitis. Systemic vasculitides: current status and perspectives. Cham: Springer International Publishing, 2016:159-71.

25. Heijl C, Harper L, Flossmann O, et al. Incidence of malignancy in patients treated for antineutrophil cytoplasm antibody-associated vasculitis: follow-up data from European Vasculitis Study Group clinical trials. Ann Rheum Dis 2011;70:1415-21.

26. Westman K, Flossmann O, Gregorini G. The long-term outcomes of systemic vasculitis. Nephrol Dial Transplant 2015;30:i60-6. 